\title{
Adaptive Optics and NICMOS Uniqueness Space
}

\author{
C. Max
}

\section{March 22, 1999}

U.S. Department of Energy

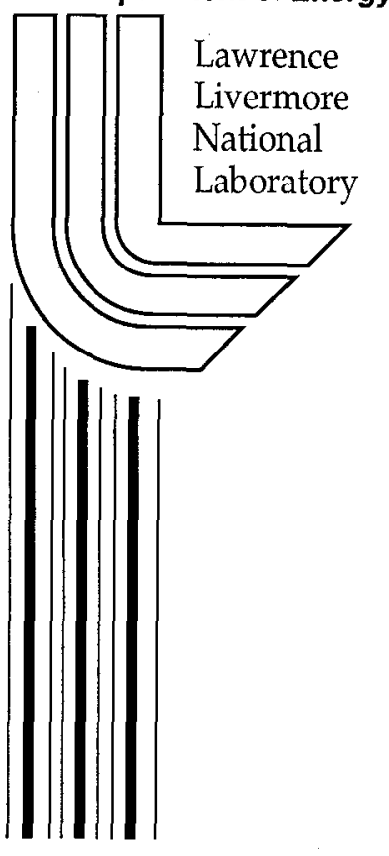




\section{DISCLAIMER}

This document was prepared as an account of work sponsored by an agency of the United States Government. Neither the United States Government nor the University of California nor any of their employees, makes any warranty, express or implied, or assumes any legal liability or responsibility for the accuracy, completeness, or usefulness of any information, apparatus, product, or process disclosed, or represents that its use would not infringe privately owned rights. Reference herein to any specific commercial product, process, or service by trade name, trademark, manufacturer, or otherwise, does not necessarily constitute or imply its endorsement, recommendation, or favoring by the United States Government or the University of California. The views and opinions of authors expressed herein do not necessarily state or reflect those of the United States Government or the University of California, and shall not be used for advertising or product endorsement purposes.

Work performed under the auspices of the U. S. Department of Energy by the University of California Lawrence Livermore National Laboratory under Contract W-7405-Eng-48.

This report has been reproduced

directly from the best available copy.

Available to DOE and DOE contractors from the

Office of Scientific and Technical Information

P.O. Box 62, Oak Ridge, TN 37831

Prices available from (423) 576-8401

http://apollo.osti.gov/bridge/

Available to the public from the

National Technical Information Service

U.S. Department of Commerce

5285 Port Royal Rd.,

Springfield, VA 22161

http://www.ntis.gov/

OR

Lawrence Livermore National Laboratory

Technical Information Department's Digital Library

http://www.llnl.gov/tid/Library.html 


\title{
Adaptive Optics and NICMOS Uniqueness Space
}

\author{
Claire Max \\ Lawrence Livermore National Laboratory
}

\section{Introduction and overview}

As part of the HST Second Decade Study a subgroup consisting of Claire Max, James Beletic, Donald McCarthy, and Keith Noll has analyzed the expected performance of near-infra-red adaptive optics systems on the new generation of 8-10 meter groundbased telescopes, for comparison with HST. In addition the subgroup has polled the adaptive optics community regarding expected adaptive optics performance over the coming five years. Responses have been received from representatives of most of the major telescopes: Gemini, VLT, Keck, LBT, and the MMT, as well as of several operational 3-4 meter telescope AO systems. The present document outlines the conclusions to date, with emphasis on aspects relevant to the NICMOS cryocooler Independent Science Review.

In general the near-infra-red capabilities of the new ground-based adaptive optics systems will be complementary to the capabilities of NICMOS. For example NICMOS will have greater $\mathrm{H}$-band sensitivity, broader wavelength coverage, and higher pointspread-function stability, whereas ground-based adaptive optics instruments will have higher spatial and spectral resolution.

Section II of this report outlines the operational constraints faced by the first generation of adaptive optics (AO) systems on new 8-10 meter telescopes. Section III describes the areas of relative strength of near-infra-red observing from the ground via adaptive optics, compared with NICMOS. A Table gives an overview of the main strengths and weaknesses of these current-generation systems. Section IV gives an indication of ground-based capabilities anticipated in the near future and in five to ten years. Section $\mathrm{V}$ contains a summary and conclusions.

\section{Operational constraints of AO systems}

Although adaptive optics on $8-10 \mathrm{~m}$ telescopes has many advantages (see Section III), it also has significant operational constraints.

\section{a) sky coverage fraction}

Adaptive optics requires a point-like reference source in order to measure atmospheric aberrations. For natural guide star $\mathrm{AO}$ this reference star must be relatively bright, typically in the range $m_{V}$ or $m_{R}=12-15$. In addition the reference star must be within the so-called "isoplanatic angle" of the astronomical object being observed. While there is not yet an exhaustive set of measurements of the isoplanatic angle, existing literature ${ }^{1,2,3}$ suggests that the isoplanatic angle ranges from $20-40$ arc sec in the nearinfra-red. This means that the probability of finding a bright enough natural reference star sufficiently near a specific (faint) astronomical object is quite small, typically well less than $10 \%$ in the near-infra-red. 
Because the sky coverage fraction for natural guide star adaptive optics is so low, most observatories with 8-10 m telescopes have made plans for developing laser guide star systems. A laser guide star can be used to measure high-order aberrations due to atmospheric turbulence, and can be pointed to arbitrary positions on the sky. A natural star is still needed to measure the centroid motion (tip-tilt), because the laser beam makes a round-trip through the atmosphere and suffers different centroid motion than an astronomical target. Tip-tilt reference stars can be as faint as $m_{V}$ or $m_{R}=17-19$. Laser guide star adaptive optics at $\mathrm{K}$ band has a coverage fraction of $50 \%$ or greater averaged over the sky, and $>10 \%$ at the Galactic Pole.

Figure 1 illustrates the sky coverage fractions calculated for adaptive optics on the Keck Telescope (Reference 1).

Note that the sky coverage fraction of $>50 \%$ is a statistical statement. Laser guide star $\mathrm{AO}$ will not work well unless there is a $17-19^{\text {th }}$ magnitude tip-tilt reference star within $20-40$ arc sec of the specific astronomical target being studied. For a given astronomical target, about half the time this condition will not be met.

The sky coverage fraction for NICMOS is virtually $100 \%$.

\section{b) field of view}

The adaptive optics field of view (the area over which the correction is close to diffraction-limited) is constrained by the isoplanatic angle, typically $20-40$ arc sec in the near-infra-red. Additionally if the diffraction limit is Nyquist-sampled, the practical field of view is determined by the size of the infra-red detector array, currently $1024^{2}$ pixels ( $\mathrm{InSb}$ and $\mathrm{HgCdTe}$ ). Thus the edge-to-edge angular distance on the array is $\sim 10$ arc sec for a 10-m telescope Nyquist-sampled at 1 micron, and $~ 30$ arc sec for an 8-m telescope at 2.2 microns. The new generation of $2048^{2} \mathrm{HgCdTe}$ arrays will double these angular distances, for the first time making the array size larger than the isoplanatic angle in the near-infra-red. The edge-to-edge field of view of NICMOS is 11, 19, and 50 arc sec for the NIC1, NIC2, and NIC3 cameras respectively.

\section{c) variable point spread function}

The strength of atmospheric turbulence is highly variable on timescales of minutes to hours. Adaptive optics systems being built today for $8-10 \mathrm{~m}$ telescopes are not able to maintain a constant point-spread function (PSF) in the face of significant variations in turbulence strength. As a consequence the PSF will vary during the night, and during an integration time. Methods exist for estimating the PSF based on measurements from the adaptive optics system itself, but the with the current state of the art the PSF for adaptive optics is neither as well-known nor as stable as that for NICMOS.

\section{d) wavelength coverage}

Ground-based telescopes can only see through atmospheric "windows" where atmospheric absorption and emission are tolerably weak. This limits wavelength coverage to the I, J, H, K, L, and M bands. On the positive side, however, InSb detectors currently used on the ground are sensitive all the way to 5 microns, and new $\mathrm{HgCdTe}$ detectors will be sensitive to 5 microns or more. NICMOS can observe at wavelengths 
Figure 1. Keck $10 \mathrm{~m}$ telescope. Strehl ratio as a function of sky coverage fraction, for laser guide star and natural guide star adaptive optics at observing wavelengths of $1.25,2.2$, and $5 \mu \mathrm{m}$. An adaptive optics system with 271 degrees of freedom is assumed. A 5 - watt CW laser forms the sodium laser guide star. The results are shown for Galactic latitudes of $30^{\circ}$ (dashed lines) and $90^{\circ}$ (solid lines).

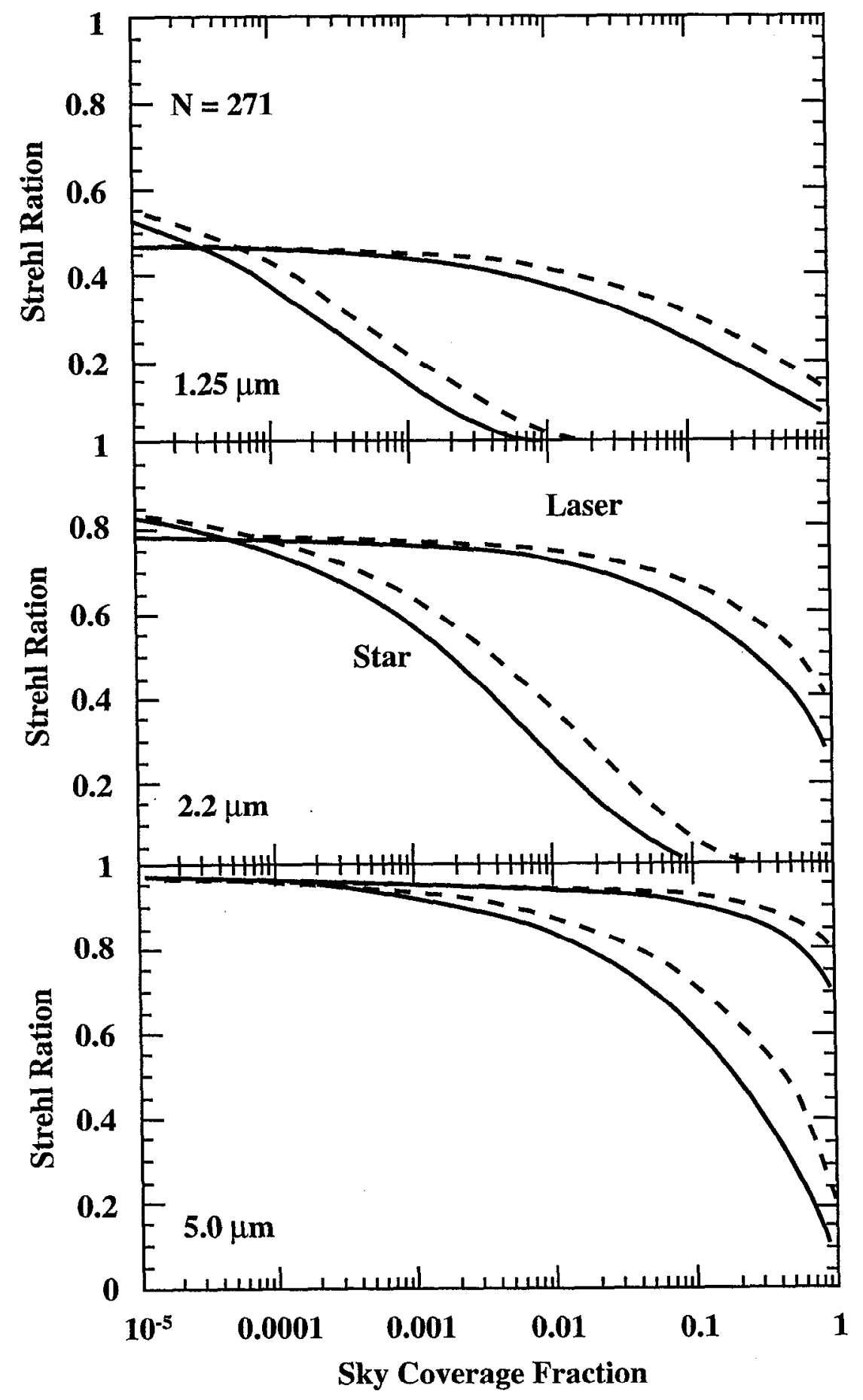


which do not penetrate the atmosphere. However the NICMOS infra-red array is only sensitive out to 2.5 microns.

\section{e) backgrounds}

$\mathrm{OH}$ in the earth's atmosphere emits strongly in the near-infra-red, causing elevated sky background noise for ground-based telescopes at wavelengths less than 2 microns. At wavelengths of 2 microns or longer, thermal emission from optics within the $\mathrm{AO}$ system itself can cause an elevated thermal background level. Background levels for NICMOS at $\mathrm{J}$ and $\mathrm{H}$ band are significantly lower than on the ground.

\section{Strengths of ground-based telescopes with adaptive optics}

Near-infra-red adaptive optics on $8-10 \mathrm{~m}$ ground-based telescopes has two areas of significant advantage, compared with NICMOS.

\section{a) Spatial resolution}

The diffraction limit of the new generation of $8-10 \mathrm{~m}$ ground-based telescopes is three to four times better than HST, simply because of the larger telescope diameter. Adaptive optics systems currently being built for these telescopes are predicted to make them diffraction-limited in the near-infra-red. In good seeing, Strehl ratios are predicted to be better than 0.3 at $\mathrm{J}$ band, and better than 0.6 at $\mathrm{K}$ band. For astronomical targets having interesting spatial structure on scales smaller than 0.1 arc sec, groundbased imaging will bring major new capabilities.

As an example of the qualitatively new information accessible at spatial resolutions of 0.02 arc sec with the Keck Telescope, Figure 2c shows images of Saturn's moon Titan taken at a wavelength of 2.1 microns. The very high spatial resolution in this image was obtained via speckle imaging over a period of several hours, followed by a month of computationally intensive data reduction. Adaptive optics will permit images such at this to be obtained in a few minutes.

Figure 2. Titan (Saturn's Largest Moon)

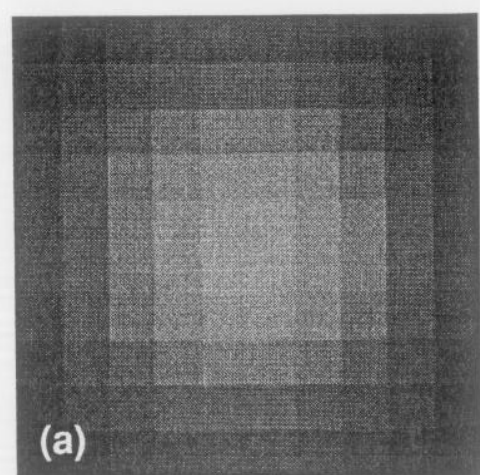

a) Conventional telescope image at a wavelength of 2.1 microns, under very good conditions (0.5 arc sec seeing).

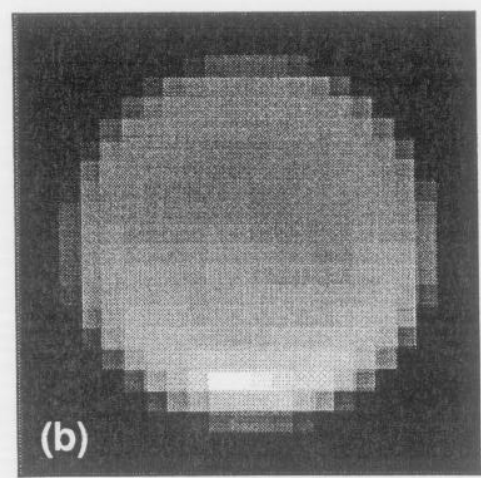

b) Hubble Space Telescope image at a wavelength of 0.85 micron (Ref. 4).

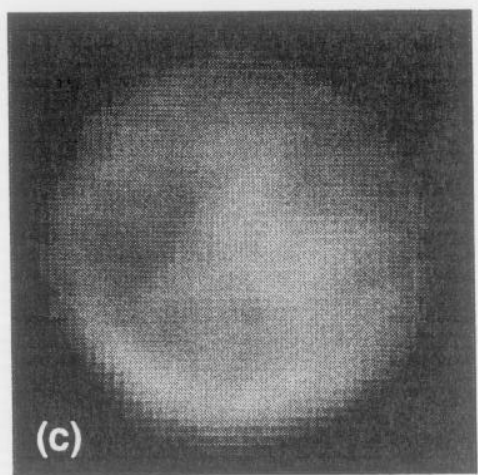

c) Diffraction-limited image from the Keck Telescope at a wavelength of 2.1 microns (Ref. 5). 
The excellent spatial resolution predicted for adaptive optics on $8-10 \mathrm{~m}$ telescopes is being realized today: the Keck II AO system is currently undergoing commissioning runs. It demonstrated a FWHM of 0.04 arc sec at $\mathrm{H}$ band on its second night of operation, with a Strehl ratio of $>30 \%$. This is illustrated in Figure 3 below.

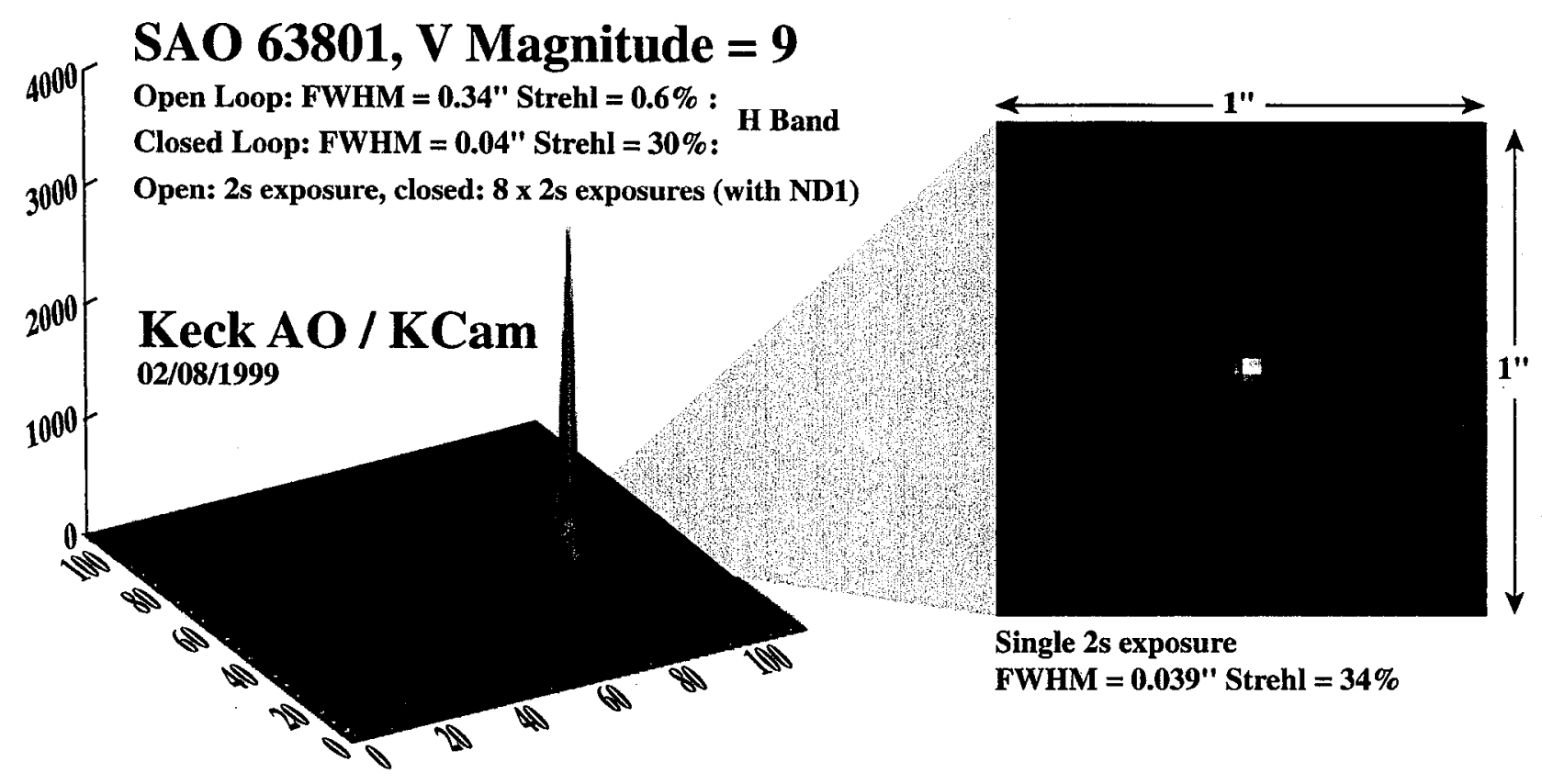

\section{b) spectroscopy}

Moderate to high spectral resolution spectroscopy on the new $8-10 \mathrm{~m}$ ground-based telescopes will be superior to HST's capabilities, which at present are limited to slit-less grism spectroscopy at a spectral resolution of 175 . Most of the new ground-based telescopes are building highly capable infra-red spectrographs. The ability to place the latter behind $\mathrm{AO}$ systems allows them to use $0.1-0.2$ arc sec slits, and to take advantage of spatial resolutions limited only by the pixel scale of the detector. In general the spectral resolution of these ground-based instruments ranges from a few thousand to 100,000, depending on the instrument and the mode of operation.

The astronomical utility of the ground-based spectrographs will be highly complementary to that of the NICMOS grisms or to future high-throughput but lowspectral-resolution spectroscopy on WFC3. For example an adaptive optics-fed integral field infra-red spectrograph would allow mapping of the internal kinematics of distant galaxies, and would have sensitivity comparable with that of HST by utilizing the gaps between the atmospheric $\mathrm{OH}$ emission lines.

Table 1 summarizes the relative strengths of ground-based $\mathrm{AO}$ on $8-10 \mathrm{~m}$ telescopes, and of NICMOS. 
Table 1

Comparison bet. NICMOS on HST and near-IR adaptive optics on 8-10 meter telescopes

\begin{tabular}{|c|c|c|}
\hline Attribute & Adaptive optics capability & NICMOS capability \\
\hline $\begin{array}{l}\text { Spatial resolution } \\
\qquad(\lambda / D)\end{array}$ & $\begin{array}{l}0.02-0.03 \text { arc sec at } 1 \mu \mathrm{m}, \\
0.04-0.05 \text { arc sec at } 2 \mu \mathrm{m}\end{array}$ & $\begin{array}{l}0.08 \text { arc sec at } 1 \mu \mathrm{m}, \\
0.17 \text { arc sec at } 2 \mu \mathrm{m}\end{array}$ \\
\hline $\begin{array}{l}\text { Ratio of light in } \\
\text { diffraction-limited core } \\
\text { to light in halo }\end{array}$ & $\begin{array}{l}\sim 30 \% \text { at } 1 \text { micron, } \\
\sim 60 \% \text { at } 2 \text { microns }\end{array}$ & $90 \%$ \\
\hline $\begin{array}{l}\text { Field of view } \\
\text { for "diffraction-limited" } \\
\text { observations (1) }\end{array}$ & $\begin{array}{l}20-40 \text { arc sec (iso. angle); } \\
10-30 \text { arc sec (Itd. by IR array) }\end{array}$ & $\begin{array}{c}11 \text { arc sec at } 1 \mu \mathrm{m}(\text { NIC } 1) \\
19 \text { arc sec at } 1.75 \mu \mathrm{m}(\mathrm{NIC} 2) \\
50 \operatorname{arc~sec}(\text { NIC } 3)\end{array}$ \\
\hline Point spread function & $\begin{array}{l}\text { Nonuniform over field of view, } \\
\text { variable in time }\end{array}$ & $\begin{array}{l}\text { Uniform over field of view, } \\
\text { steady in time to } 1-2 \%\end{array}$ \\
\hline Photometric accuracy & Not yet well understood & $\begin{array}{c}\text { Absolute accuracy }<5 \% \text {, } \\
\text { relative accuracy } 1-2 \%\end{array}$ \\
\hline Sensitivity & Better in $\mathrm{K}$ band ? & Better in $\mathrm{J}$ and $\mathrm{H}$ bands $(<1.9 \mu \mathrm{m})$ \\
\hline Wavelength coverage & $\begin{array}{l}\text { Only in atmospheric windows, } \\
\text { but reaches to } 5 \mu \mathrm{m}\end{array}$ & $0.8-2.5 \mu \mathrm{m}$ \\
\hline $\begin{array}{l}\text { Spectroscopy } \\
\text { capability }\end{array}$ & $\begin{array}{c}\text { Spectral resolution } R=25,000 \\
\text { near term; } R=100,000 \text { in } 5 \text { yrs. } \\
\text { Slit width }<0.2 \text { arc sec }\end{array}$ & $\begin{array}{l}\text { Spectral resolution } R=175 . \\
\text { No slit. }\end{array}$ \\
\hline Sky coverage fraction & $\begin{array}{c}<10 \% \text { for natural guide star, } \\
<50 \% \text { for laser guide star }\end{array}$ & $100 \%$ \\
\hline
\end{tabular}

Note (1) For this discussion, "diffraction-limited" observations refers to core:halo energy ratio greater than 0.3 


\section{Section IV. Ground-based capabilities now and in 5-10 years}

In this section we attempt to predict what the state of the art will be in five to 10 years. As with all fast-moving technologies there are likely to be surprises.

\section{a) natural guide star adaptive optics systems on 8-10 m telescopes}

Virtually all of the new generation of $8-10 \mathrm{~m}$ ground-based telescopes plan to have AO capabilities within a few years. The Keck II system is currently being tested at the telescope. A curvature-sensing AO system will be deployed at Gemini North's first light. Within 3-4 years all of the large telescopes will have operating natural guide star AO systems. These systems have all been designed for operation in the near-infra-red; they vary from 36 to several hundred degrees of freedom.

In five years, methods to determine what the point spread function was during an observation will have been refined. Although the PSF will still vary as atmospheric conditions change, it will be possible to determine to high accuracy what the PSF was. The field of view of ground-based near-infra-red imagers will be increased during the coming five years, because of the availability of $2048^{2} \mathrm{HgCdTe}$ detectors. The natural guide star sky coverage will remain relatively modest, at $10 \%$ or less.

In ten years many of the large-telescope AO systems will have been upgraded to control more degrees of freedom. High-quality correction will extend to shorter wavelengths (first to I and R band, and then to shorter wavelengths). Strehl ratios in the near-infrared will be above $80 \%$ most of the time. The PSF will vary, but will be accurately known and very well calibrated. Methods will have been developed to extend the isoplanatic patch to one or two arc minutes.

\section{b) laser guide star systems on $8-10 \mathrm{~m}$ telescopes}

Deployment of laser guide stars on 8-10 m telescopes is lagging behind deployment of $\mathrm{AO}$ systems. This is partly due to the desire to make the natural guide star systems work robustly prior to adding the complications of a laser, and partly due to the current status of laser technology.

As a result, we do not foresee routine laser guide star operation on any $8-10 \mathrm{~m}$ telescope until one or two years from now. We think it will take three to four years for laser guide stars to come into broad use on the largest telescopes. Hence there is a several-year period when the sky coverage for ground-based $\mathrm{AO}$ systems will be quite limited, and NICMOS will have most of the playing field to itself.

In ten years lasers will be in broad use, and methods will have been developed to use multiple guide stars in order to ameliorate the so-called "cone effect." Advanced techniques will reduce the need for a close-in tip-tilt star, raising the laser guide star sky coverage fraction to $100 \%$. 


\section{Summary and conclusions}

The roles of NICMOS on HST and of near-infra-red adaptive optics on the ground will be strongly complementary. NICMOS will have higher sensitivity at $\mathrm{J}$ and $\mathrm{H}$ bands, will do more accurate photometry, and will have higher sky coverage. Adaptive optics on $8-10 \mathrm{~m}$ ground-based telescopes will excel at spectroscopy, will have higher spatial resolution, and may have better sensitivity at $\mathrm{K}$ band.

Two examples illustrate how the relative strengths of ground and space will be exploited.

1) NICMOS will be used for the discovery, photometry, and general morphology of very distant galaxies, while ground-based $\mathrm{AO}$ will be used to study their highresolution morphology and spectroscopy.

2) Ground-based $\mathrm{AO}$ will be used to separate faint individual stars in the Galactic Center and to measure their velocity structure via proper motions and spectroscopy. NICMOS will be used to measure very faint structures near Sag $A^{*}$ with high dynamic range, and to study stellar variability due to gravitational lensing by the central black hole.

\section{References}

1. "Adaptive Optics for the Keck Observatory", G. Chanan, G. Djorgovski, A. Gleckler, S. Kulkarni, T. Mast, C. Max, J. Nelson, and P. Wizinowich, W. M. Keck Observatory Report number 208 (1994 and 1996), chapter 4.

2. "Laser guide star for 3.6- and 8-m telescopes: performance and astrophysical implications", M. le Louarn, R. Foy, N. Hubin, and M. Tallon, M.N.R.A.S. $\underline{295}, 756$ (1998).

3. "Adaptive optics sky coverage calculations for the Gemini-North telescope", B. L. Ellerbroek and D. W. Tyler, P.A.S.P. 110 165 (1998); "Rayleigh Beacon Adaptive Optics Imaging of ADS 9731: Measurements of the Isoplanatic Field of View", J. C. Christou, B. Ellerbroek, R. Q. Fugate, D. Bonaccini, and R. Stanga, Ap. J. 450, 369 (1995).

4. "Titan's surface, revealed by HST imaging", P. H. Smith, M. T. Lemmon, R. D. Lorenz, L. A. Sormovksy, J. J. Caldwell and M. D. Allison. Icarus 119336 (1996).

5. "Titan: High Resolution Speckle Images from the Keck Telescope", S. G. Gibbard, B. Macintosh, D. Gavel, C. E. Max, I. de Pater, A. M. Ghez, E. F. Young, and C. P. McKay, Icarus, in press, (1999). 